\title{
Transcriptome mechanisms underlying interaction of polystyrene nanoplastics and wheat Triticum aestivum $L$.
}

\section{Qixing Zhou ( $\nabla$ zhouqx@nankai.edu.cn )}

Nankai University

Jiapan Lian

Nankai University

Weitao Liu

Nankai University

Shuzhen Men

Nankai University https://orcid.org/0000-0001-6472-6829

Jiani Wu

Nankai University

\section{Yuebing Sun}

Key Laboratory of Original Environmental Pollution Prevention and Control, Ministry of Agriculture and Rural Affairs

\section{Aurang Zeb \\ Nankai University \\ Tianzhi Yang \\ Nankai University \\ Qiying Ma \\ Zhejiang University}

\section{Article}

Keywords: Differently Expressed Genes, Co-expression Network Analyses, Ambient Polystyrene Nanoparticles, KEGG Pathways

Posted Date: October 30th, 2020

DOI: https://doi.org/10.21203/rs.3.rs-98748/v1

License: (c) (i) This work is licensed under a Creative Commons Attribution 4.0 International License. Read Full License 


\section{Abstract}

Microplastics (MPs) and nanoplastics (NPs) have inevitably entered and accumulated in terrestrial ecosystems. Recently, the report on MPs/NPs taken-up by crop plants has raised particular concerns. However, molecular mechanisms underlying the interaction of MPs/NPs and crop plant are still vague and elusive. To address this scientific gap, we identified differentially expressed genes (DEGs) and performed co-expression network analyses in hydroponically grown wheat (Triticum aestivum L.) with ambient polystyrene NPs (PSNPs) concentration (0-10 mg/L). PSNPs could significantly shape the gene expression pattern in wheat with a tissue-specific mode. Four regulatory modules associated with the plant performance and nutrient capture were identified using WGCNA analysis. In addition, carbon metabolism, amino acid biosynthesis, MAPK signaling pathway-plant, plant hormone signal transduction, and plant-pathogen interaction were the most enriched KEGG pathways for all DEGs and target module eigengenes. These results confirm that NPs-induced genetic changes are the dominating driven forces for the observed plant growth or defense responses, shedding new light on the molecular mechanism and environmental implication behind the interaction of NPs and crop plants.

\section{Introduction}

Plastic has become increasingly ubiquitous on our planet with $79 \%$ of the total plastic production (up to 65.6 billion tones) ending up in landfills or the natural environment ${ }^{1,2}$. In the last two decades, both microplastics (MPs, $1 \mu \mathrm{m}$ to $5 \mathrm{~mm}$ ) and nanoplastic (NPs, $<1 \mu \mathrm{m})$ in marine, freshwater, and terrestrial ecosystems have attracted widespread attention all over the world $3,4,5,6$. Compared with the welldocumented reports of plastic debris in aquatic ecosystems, there is a marked lack of knowledge about the impact of MPs/NPs on terrestrial ecosystems (especially agroecosystems), which has become a field of interest for scientists $3,7,8$.

As reported, MPs/NPs can enter the agricultural environment through sewage irrigation $9,10,11$, sludge and fertilizer application $12,13,14$, plastic-film mulch degradation ${ }^{15,16}$, and even atmospheric deposition 17, 18. Consequently, the mass input loadings of plastic debris in agroecosystems have led to the frequent occurrence of MPs/NPs in agricultural soil like arable soil of southwestern China ${ }^{19}$, Swiss floodplain soils ${ }^{20}$, and farmland of southeast Germany ${ }^{21}$. The other possible formation of NPs during biosolids treatment and daily processes has been alerted ${ }^{13,22}$, albeit there is no direct evidence of their existence in the agricultural environment. Considering the supporting role of agricultural ecosystems for human survival, any potential adverse effects or unknown consequences of MPs/NPs for environmental and human health should be better understood ${ }^{3,23}$.

Owing to the limitation of detection techniques and complex plant barriers, MPs were once considered unable to enter the plant and it was also doubtful whether plants could absorb NPs ${ }^{24,25}$. Until recently, the plant uptake of NPs has been confirmed through multiple advanced methods including the combined application of fluorescent labeling and electron microscopy, as well as the innovative synthesis of 
palladium-doped NPs $26,27,28$. Accumulation of MPs/NPs in plants might induce phytotoxicity and an active defense reaction, raise the food safety concerns and pose the ecological risk ${ }^{29}$. However, the existing conclusions of MPs/NPs on crop phytotoxicity seems to be contradictory. For example, exposure to NPs could inhibit growth or mediate oxidative damage in rice ${ }^{30,31}$, Vicia faba ${ }^{32}$, ryegrass ${ }^{33}$, spring onion ${ }^{34}$, and Arabidopsis thaliana ${ }^{35}$. Inversely, our previous studies showed that PSNPs had low phototoxicity and could dramatically enhance the growth performance of wheat when exposed to ambient concentrations ${ }^{27}$. In most cases, the effects produced by nanomaterials are unpredictable and largely inconsistent in different studies, which relied on many factors such as particle size, exposure time, concentration, plant species, and growth medium ${ }^{36}$. Besides, some great works demonstrated the evolution and adaption strategies of higher plants to NPs ${ }^{37,38}$, while the underlying molecular mechanism remains largely unclear.

Nanoparticle-specific changes in gene expression were vigorously investigated in many plant-metal NPs interactions studies, which made it possible for us to know how plants and plastic interact ${ }^{39,40}$. To our knowledge, although most of the peer-reviewed works explored the interactions of NPs with plants, gene characteristics behind plant traits or defense reactions are still unavailable. Herein, we hypothesized that the genetic changes in wheat caused by the environmentally-relevant level of PSNPs could be responsible for the observed interaction effects. The present study thus aims at verifying the gene expression variation in wheat shoots and roots, linking the observed physio-biochemical indicators with modules to identify functional genes by a WGCNA network analysis. Together, the results presented here provide a molecular mechanism of NPs and plant interaction, and point out some promising directions for future research.

\section{Materials And Methods}

\section{Seeds and Chemicals}

Wheat seeds (Xiaoyan 22) were purchased from Northwest Agriculture \& Forestry University. To avoid NPs pollution, seeds were stored in a sealed kraft paper bag at $4-8{ }^{\circ} \mathrm{C}$ before use. Polystyrene nanoplastics (PSNPs) were purchased from Huge Biotechnology Co., Ltd (Shanghai, China). Morphology, particle size, composition corroboration, Zeta potential, and hydrodynamic diameter of PSNPs had been previously characterized and described in our early-stage work ${ }^{27}$. Briefly, the PSNPs resemble spheres with smooth surface morphology. The average particle diameter of PSNPs is $87.8 \pm 8.6 \mathrm{~nm}$ with no surface functional groups. Additionally, all other commercial chemicals were purchased from Maclin Biochemical Technology Co., Ltd (Shanghai, China).

\section{Plant Cultivation and Exposure}

Wheat seeds were surface sterilized with 2.0\% $(\mathrm{v} / \mathrm{V})$ hydrogen peroxide $\left(\mathrm{H}_{2} \mathrm{O}_{2}\right)$ for $30 \mathrm{~min}$ and rinsed thoroughly with deionized water. Then these seeds were germinated at $25^{\circ} \mathrm{C}$ in a dark incubator for five days. Healthy wheat seedlings were selected and hydroponically cultured for one week in $25 \%$ strength 
Hoagland solution. The exposure solutions were obtained by adding dialyzed PSNPs stock solution into freshly prepared $25 \%$ strength nutrient solution and then dispersed by ultrasound ( $100 \mathrm{~W}, 25 \mathrm{kHz})$ for 10 min. Subsequently, uniform plantlets were chosen and transplanted into a beaker containing $500 \mathrm{~mL}$ of PSNPs exposure solution at the ambient concentrations of $0.01,0.1,1$, and $10 \mathrm{mg} / \mathrm{L}$. All the beakers were wrapped with tin foil to maintain a dark environment and the solutions were replenished with the fresh nutrient solution every three days. These seedlings subjected to PSNPs were cultivated in a growth chamber with a day/night temperature of $28^{\circ} \mathrm{C} / 20^{\circ} \mathrm{C}, 60 \%$ relative humidity, $14 \mathrm{~h}$ photoperiod, and a light intensity of 6000 lux for three weeks. Four replicates were set for each treatment. At harvest time, wheat seedlings were gently removed from the culture solution and thoroughly washed with tap water followed by deionized water. The youngest fully developed leaves and roots were collected and immediately snap-frozen in liquid nitrogen for RNA extraction. The dry weights of roots and shoots were recorded after $48 \mathrm{~h}$ vacuum freeze-drying.

\section{Total RNA Isolation and Libraries Construction}

Total RNA was extracted from the leaves or roots samples by using TRIzol reagent (Invitrogen, Carlsbad, $\mathrm{CA}$ ) following the manufacturer's instructions. Possible RNA degradation and contamination were estimated using $1 \%$ agarose gel electrophoresis. The concentration and purity of the total RNA were determined with a NanoDrop 2000 spectrophotometer (Thermo Scientific, MA, USA). Also, the resulting RNA integrity was confirmed by using the Agilent 2100 Bioanalyzer system (Agilent Technologies, Santa Clara, CA).

The total RNA samples from four biological replicates in each treatment were sent to Novogene Bioinformatics Technology Co., Ltd (Beijing, China) for commercial Illumina sequencing. Sequencing libraries were established on the Illumina HiSeq 2500 platform (Illumina, San Diego, USA) and 150 bp paired-end reads were generated. Briefly, Poly (A) mRNA was isolated from total RNA using poly-T oligo-

attached magnetic beads, after which mRNA was broken up into short fragments in NEBNext First Strand Synthesis Reaction Buffer. Using these short chains as templates, the first-strand cDNA was synthesized with random hexamer primer and M-MuLV Reverse Transcriptase (RNase $\mathrm{H}-$ ). Subsequently, the secondstrand cDNA synthesis was performed using DNA Polymerase I and RNase $\mathrm{H}$. The double-strand cDNA fragments were purified, adenylated for end-repair, adding a poly (A), and ligated to NEBNext adapters. After purification, the cDNA fragments with a preferential length of 205-300 bp were selected for PCR. Finally, the amplified PCR products were purified (AMPure XP system) and library quality was assessed on the Agilent Bioanalyzer 2100 system platform.

\section{mRNA sequence data processing}

To ensure the quality and reliability of raw data, the clean reads were obtained by removing reads containing adapter, reads containing ploy-N (unknown sequences), and low-quality reads. Meanwhile, Q20, Q30, and GC contents of the clean data were measured and all downstream analyses were consequently based on the resulting clean reads. The paired-end clean reads were aligned to the wheat reference genome (ftp://ftp.ensemblgenomes.org/pub/release-23/plants/fasta/triticum_aestivum/dna/) 
using TopHat v2.0.12. After mapping, HTSeq v0.6.1 was employed to count the reads numbers that were aligned to each unigene. Given that the effects of sequencing depth and gene length for the reads count at the same time, the gene expression level was calculated using FPKM value (Fragments Per Kilobase of transcript sequence per Million base pairs sequence) ${ }^{41}$. After normalizing and transforming the expression level of each gene over the control, differentially expressed genes (DEGs) were identified by pairwise comparisons using DESeq2 R package (1.16.1) with an absolute value of log2 fold change (FC) 31 and a false discovery rate (FDR) $£ 0.05$, in which the resulting $p$-values were adjusted using the Benjamini \& Hochberg's approach.

\section{Weighted Gene Co-expression Network Analysis (WGCNA)}

Due to the large genome of wheat species, all genes were filtered with an FPKM value of 0.5 as the threshold to obtain a suitable genome size before network operation. After removing non-varying or lowabundance genes, 40,411 genes with averaged FPKM in wheat tissues were chosen for the unsigned coexpression network analysis using the WGCNA package in $\mathrm{R}^{42}$. According to the approximate scale-free topology preconditions, an adjacency matrix was constructed using a soft threshold power of 7 and the corresponding dissimilarity of 0.2 . Then, network modules of eigengenes were identified based on the Dynamic Tree Cut algorithm with a cut-off of ${ }^{3} 30$ genes and a merging cut height of 0.25 . To identify functional modules in a co-expression network, the module-trait relationships between modules agronomic traits were calculated, and the correlated modules with statistical significance $(p £ 0.05)$ and Pearson's correlation coefficient $\left(r^{3} 0.5\right)$ were retained for further analysis. In detail, the impact of PSNPs on agronomic characteristics (SPAD, Pn, Gs, Tr), mineral nutrients ( $\mathrm{Cu}, \mathrm{Mn}, \mathrm{Zn}, \mathrm{Fe}, \mathrm{Mg}$ ), and metabolites of wheat seedlings were clearly illustrated in our previous work ${ }^{27}$. Hub genes within the functional modules were identified topologically by calculating intra-modular connectivity. Eventually, the top 20 hub genes within a module were highly marked and their mutual interaction networks were visualized using Cytoscape v.3.7.2.

\section{GO and KEGG Enrichment Analysis}

All DEGs and the eigengenes of each extracted module were subsequently subjected to the enrichment analysis for Gene Ontology (GO) and KEGG (Kyoto Encyclopedia of Genes and Genomes) pathways, respectively. Significantly enriched GO terms and KEGG pathways were identified with a corrected $p$-value less than 0.01 .

\section{Validation of RNA-Seq by RT-qPCR}

To verify the performance of total RNA sequencing, all RNA samples were further used to confirm the transcript results using reverse-transcription real-time PCR (RT-qPCR). The RT-qPCR assay was performed on CFX96 Real-Time PCR Detection System (Bio-Rad, Hercules, USA) using a One-Step qRT-PCR Kit (Toyobo, Osaka, Japan) according to the manufacturer's instructions. Target-specific PCR primers designed with Primer Premier 5.0 software were presented in Table S1, in which Wheat $\beta$-actin (forward: GGAATCCATGAGACCACCTAC; reverse: GACCCAGACAACTCGCAAC) was used as an internal reference. 
The reactions were conducted in a $20 \mu \mathrm{L}$ volume containing $10 \mathrm{~mL}$ SYBRß Green Supermix, $0.4 \mathrm{~mL}$ each primer, $1 \mathrm{~mL} \mathrm{Mn(OAc)})_{2}, 50 \mathrm{ng}$ template cDNA under the following conditions: $95^{\circ} \mathrm{C}$ for $30 \mathrm{~s}, 61^{\circ} \mathrm{C}$ for 20 $\min , 95^{\circ} \mathrm{C}$ for $30 \mathrm{~s}$, followed by 43 cycles of $95^{\circ} \mathrm{C}$ for $15 \mathrm{~s}, 55^{\circ} \mathrm{C}$ for $10 \mathrm{~s}$ and $74{ }^{\circ} \mathrm{C}$ for $15 \mathrm{~s}$, and the last step at $72{ }^{\circ} \mathrm{C}$ for $5 \mathrm{~min}$. Three technical replicates were performed in all qRT-PCR experiments, and the relative gene expression was independently calculated.

\section{Data availability}

The data that support the findings of this study are available from the corresponding author on reasonable request.

\section{Results}

\section{Identification of DEGs involved in PSNPs treatments}

Differentially expressed genes (DEGs) in wheat shoots and roots were identified by comparing the expression level of each gene in PSNPs treatments and the control group (Fig. 1a; Fig. S1). As shown in Fig. 1c, the number of differentially up-regulated or down-regulated genes in the shoots and roots of wheat steadily increased with the increase of PSNPs exposure concentration. At the same time, the number of genes down-regulated was almost twice that of up-regulated genes in wheat tissues. The most interesting aspect of Fig. 1c was that regardless of the PSNPs exposure concentration, the number of down-regulated genes in the roots is always higher than that in the shoots. Additionally, the Venn diagram further illustrates the possible DEGs relations among different PSNPs treatments, where 1910 (7.83\%) and 1167 (6.11\%) DEGs were common to all four PSNPs concentrations in root and shoot, respectively (Fig. 1d). Taken together, these above results suggested that PSNPs did induce genetic changes in wheat as environmental xenobiotics and meanwhile down-expressed genes were the dominant part of differential genes involved in PSNPs treatments, especially in wheat root.

\section{Characterization of co-expression networks related to PSNPs-induced traits}

In order to figure out the candidate regulatory genes related to our desired traits (agronomic characters, mineral nutrients, and metabolites), all filtered transcripts from different wheat tissues were pooled together for subsequent WGCNA analysis. Accordingly, a total of eight modules were obtained in our study that were named in different colors (Fig. 2a,b). Among all the modules, the turquoise module (MEturquoise) contained the largest number of genes (5678), followed by MEblue (5286), MEbrown (4559), MEyellow (2703), MEgreen (1009), and MEred (349) (Fig. S3). It could be seen that the expression level of the module in wheat was highly tissue-specific, which was also reflected in the expression patterns of DEGs (Fig. 2b; Fig. S1). By way of example, the eigengenes in blue and green modules were 
over-expressed in the roots, while low-expressed in shoots. Similar phenomena were also found in MEturquoise and MEred. Moreover, the hierarchical cluster analysis result demonstrated that MEblue and MEgreen were provided with high homogeneity, and they appeared to be closely related (Fig. S2c).

Through correlation analysis, we identified four modules (MEblue, MEgreen, MEturquoise, MEred) that were significantly related to the target traits (SPAD, biomass, $\mathrm{Pn}, \mathrm{Gs}, \mathrm{Tr}$, and $\mathrm{Cu}, \mathrm{Mn}, \mathrm{Zn}, \mathrm{Fe}, \mathrm{Mg}$ contents) (Fig. 2c). A remarkably strong correlation was also found between these four modules and metabolites (Fig. S5). In particular, agronomic characters (SPAD, biomass, Pn, Gs, Tr) and almost all metabolites shared a common correlation trend, positively related to MEblue and MEgreen while negatively correlated with MEturquoise and MEred. However, for mineral nutrients, the results changed and depended largely on the element types. Obviously, it was worth noting that the relevance between macronutrient $(\mathrm{Mg})$ and micronutrients ( $\mathrm{Cu}, \mathrm{Mn}, \mathrm{Zn}, \mathrm{Fe}$ ) was element-dependent (Fig. 2c). In terms of eigengene expression within modules, transcripts comprising Meblue and MEgreen displayed high expression in root but low expression in leaf. Conversely, the transcripts expression trends in MEturquoise and MEred were totally opposite (Fig. S4). Overall, these results indicated that wheat gene expression profile exposed to PSNPS was tissue-specific, and functioning genes that control target traits were basically clustered in four modules, including MEblue, MEgreen, MEturquoise, and MEred.

\section{Hub genes and GO terms associated with PSNPs exposure}

To investigate the possible functions of tracked modules, we divided these four modules into two large sets where MEblue and MEgreen were regarded as the first groups, and MEturquoise and MEred were the second group. In this context, the top 20 genes were screened according to the degree of connectivity within each module for better display of the selected module co-expression network. Results of gene interaction analysis showed that TraesCS3D02G236700 and TraesCS3D02G353100 were the key genes of the blue and green modules respectively (Fig. 3a). Of note, no specific network functions of the module were identified via the hubs approach. Therefore, the genes in MEblue and MEgreen were merged for further Gene Ontology (GO) enrichment analysis, and the top $20 \mathrm{GO}$ terms were presented as well (Fig. $3 b)$. Most of the significant $\mathrm{GO}$ terms enriched by these modules were mainly nucleoside metabolic and protein processing processes, including GTPase activity (75), protein folding (53), cofactor metabolic process (63), nucleoside phosphate metabolic process (56), and purine nucleoside triphosphate metabolic process (47), etc. Other than these, some GO terms involved in metal transport were also found such as ion transmembrane transport (45), metal ion transport (47), cation transmembrane transport (30), inorganic ion transmembrane transport (30), copper ion transport (5), ion antiporter activity (5) and metal ion transmembrane transporter activity (24) (Supporting material excel_1). More importantly, the same GO terms for metal ion transport were also enriched by DEGs (Fig. S5, 6). In the case of metal ion transport, the over-expression of these eigengenes primarily occurred in wheat roots when exposed to high concentrations of PSNPs, while only a small number of genes showed the differential expression after PSNPs treatments (Fig. 3c). 
As for the negative modules, key genes in turquoise (TraesCS7A02G459700) and red module (TraesCS3A02G481500) were also screened out, which belong to the Glycoside hydrolase family 17 (Fig. $4 a, b)$. Correspondingly, the results showed that cofactor metabolic and biosynthetic processes $(75,45)$, sulfur compound metabolic process (38), amino acid metabolic and biosynthetic processes $(47,74)$, and metal cluster binding (44) were the significant GO function items (Fig. 4c). With the exception of these, it was worth noting that many other $\mathrm{GO}$ terms are related to the metabolism and biosynthesis of amino acids, including serine family amino acid, glutamine family amino acid, aspartate family amino acid, sulfur amino acid, cysteine, lysine, histidine, and amino acid activation (Supporting material excel_2). In more detail, we investigated the expression profile of these eigengenes in different wheat tissues. Interestingly, the expression of these genes in roots did not change much while there were significant expression alterations in leaves especially at $0.1 \mathrm{mg} / \mathrm{L} \mathrm{PSNPs} \mathrm{(Fig.} \mathrm{4d).} \mathrm{As} \mathrm{a} \mathrm{result,} \mathrm{it} \mathrm{was} \mathrm{reasonable} \mathrm{to}$ speculate that these two modules are mainly responsible for the biosynthesis and metabolism of amino acids.

\section{Enriched KEGG pathway in wheat exposed to PSNPs}

In addition to GO enrichment analysis, the mainly enriched biological pathways in

wheat induced by PSNPs exposure were identified by two independent KEGG analyses (Table 1). In WGCNA, there were 49(10.3\%) and 47 (8.8\%) genes in Meblue-green and Meturquoise-red module respectively that were assigned to carbon metabolism, while the most significantly enriched pathway in Meturquoise-red was ribosome. Notably, a large portion of genes in MEblue-green was matched to other

biological pathways such as biosynthesis of amino acids, oxidative phosphorylation, glycine, serine and threonine metabolism, and pentose phosphate pathway. For comparison, it was expected to distinguish differences in enriched pathways in wheat shoot and root using MetaDE analysis. However, most of the DEGs were mapped to similar KEGG terms with slightly different $p$-value, which included plant-pathogen interaction, plant hormone signal transduction, MAPK signaling pathway - plant and glycerophospholipid metabolism.

\section{Validation of the RNA-sequencing results}

In the present study, the expression of 10 genes was verified by an RT-qPCR approach to assess the performance characteristics of RNA sequencing results. As shown in Fig. 5, there was a significant correlation $(p<0.01)$ in the relative gene expression between RT-qPCR and RNA-Seq method, indicating that the transcriptome sequencing results are reliable. 


\section{Discussion}

With the continuous improvement and innovation of nanomaterial analytical techniques, more clear shreds of evidence about the uptake and internalization of MPs/NPs by plants were presented ${ }^{35,43}$. In this context, it is particularly necessary to explore the potential effects of MPs/NPs on plants, especially crops. Therefore, in the present study, a model experiment involving the real environmental concentration of NPs was implemented for hypothesis verification that internalized NPs could affect plant traits by altering gene expression profiles. Nevertheless, the PSNPs used in our study were commercial products with perfect sphericity and homogeneity that are less relevant to the bulk of NPs in the real environment, these standard NPs could be used as a temporary substitute or bridge for understanding environmental concerns or the biological effects of NPs ${ }^{28}$. Therefore, in the following, we will elucidate the possible mechanisms of plants' response to NPs and establish a potential underlying biological implication.

Micro- and nanoplastics have been proven to interfere with plant performance, whether it is aquatic or terrestrial ${ }^{31,33,37}$. But so far, there is no sufficient evidence that MPs with environmental characteristics can enter plants and finally affect plant growth. For most scenarios, some hypothesized indirect mechanisms have been proposed like alteration in soil physicochemical and hydrological properties, changes in soil microbial community and root symbionts, and so on ${ }^{3,44,45}$. In contrast, plastics with nanometer-size could be directly absorbed by plants and then exerted a significantly negative effect on plant production ${ }^{31,35}$. In the present study, we found a great improvement in wheat biomass (Fig. 1b), which might be related to enriched plant hormone signal transduction (Table 1). Similarly, both Zhou, Lu ${ }^{30}$ and Sun, Yuan ${ }^{35}$ reported that phytohormone biosynthesis (Jasmonic acid) and plant hormone signal transduction were the significantly enriched KEGG pathways induced by NPs in Arabidopsis thaliana and rice, respectively. It is well known that phytohormones are important signal molecules that regulate various aspects of plant growth, development, and even response to both biotic and abiotic stress ${ }^{46}$. For this reason, it is highly reasonable to assume that phytohormone biosynthesis and signal transduction might be responsible for the NPs-induced changes in plant growth. Hence, the signaling pathway and network regulation of plant hormone in NPs-plant interaction should be a key focus of future research.

On the other hand, the PSNPs-induced activation in carbon metabolism might also play a crucial role in wheat growth (Table 1). In our recent study, the metabolic analysis showed that exposure to PSNPs significantly regulated galactose metabolism, glyoxylate and dicarboxylate metabolism, TCA cycle, as well as starch and sucrose metabolism, collectively leading to an increase in biomass ${ }^{27}$. Consistent with our results, carbon metabolism including fatty acid metabolism and dicarboxylate metabolism was the top KEGG metabolic pathway performed on all DEGs in rice ${ }^{30}$. On the contrary, the difference observed in plant growth (carbon content \& biomass) due to carbon metabolism may be species-dependent. Overall, which species of plant could be improved or inhibited in plant biomass production by NPs is an important issue $^{3}$. 
Although the uptake and transport pathway of NPs in plants is not yet clear, it is quite likely that the NPs in the epidermal cell and catheter of the xylem could physically block the absorption of nutrients ${ }^{32,35,47}$. Consistently, the plant with nutrient limitation would allocate more biomass into the root, resulting in a lower shoot/root (S:R) ratio ${ }^{37,48}$. In our previous study, we indeed found a "blockage effect" on micronutrients uptake in PSNPs-exposed wheat while no obvious inhibition or even promotion in root macronutrient contents ${ }^{27}$. Interestingly, in this research, the discriminative expression of genes involved in metal ion transport was mainly observed in the root instead of the shoot (Fig. 3d). More specifically, these DEGs expression changes occurred in high exposure concentrations and mainly targeted at the transmembrane transport of nutrients (transmembrane transporters \& transporter activity). It has been found that 1) the absorption and transportation of $\mathrm{Mg}^{2+}$ in wheat roots are non-selective and stable ${ }^{49}, 2$ ) $\mathrm{Mg}^{2+}$ reached the endodermis through the apoplast pathway faster than the symplast pathway ${ }^{50}$, and 3 ) the transport of nutrient elements (zinc, iron, and copper) in plant root cells is mainly based on ionophores and cotransporters ${ }^{51}$. Despite many studies have suggested that NPs penetrate into the xylem via the apoplastic pathway, the internalized PSNPs seem to promote transpiration pull to a large extend ${ }^{27,43}$. Taken together, this implies that the absorbed NPs through the water transport system have little effect on $\mathrm{Mg}^{2+}$ transport, which might be offset by increased water flow in roots. But the targeted genes associated with transmembrane transport or transporter activity of micronutrients in roots were upregulated under insufficient trace elements, thus indicating that they may be a genetic response for promoting the symplast transport and xylem loading processes of trace elements. Notably, an important question is whether this phenomenon occurs only in presence of PSNPs. Otherwise, NPs in agricultural ecosystems might reduce the quality of crops to a greater extent.

Besides the above, plant-pathogen interaction is another significantly enriched KEGG pathway in wheat shoot and root. This is highly consistent with the result of Sun, Yuan ${ }^{35}$, of which plant-pathogen interaction KEGG pathway is commonly found in Arabidopsis thaliana when exposed to two functionalized PSNPs (PS-SO ${ }_{3} \mathrm{H} \& \mathrm{PS}-\mathrm{NH}_{2}$ ). Both the downregulation of genes associated with pathogen in shoot and root demonstrated that NPs exposure could decrease plant disease resistance. Meanwhile, the upregulated amino acid biosynthesis pathway and increased amino acids level were actually observed in wheat shoot, which might a tolerant response to pathogen infection ${ }^{52}$. With regard to NPs taken-up, excess mucilage and exudates production (mainly low molecular organic acids: LMOA) associated with amino acid biosynthesis might play a crucial role in the first barrier defense ${ }^{53}$. Moreover, Serval reports showed the phytotoxicity of NPs to higher plant due to the overproduction of ROS 30,35, which may induce the anti-stress KEGG pathway such as MAPK signaling pathway-plant and glycerophospholipid metabolism. However, low phytotoxicity of NPs to wheat was determined in our previous study, so MAPK signaling pathway-plant was probably triggered by biotic stress stimuli such as pathogen infection. These results implied that NPs could potentially regulate the expression of disease resistance functional genes and thus reduce the disease resistance of crop plants.

\section{Conclusions}


In summary, correlative transcriptome analysis based on differentially expressed genes (DEGs) and weighted gene co-expression network (WGCNA) revealed important molecular evidence and mechanisms of wheat exposure to NPs. In the present study, we found a tissue-specific pattern of DEG expression in wheat when exposed to ambient PSNPs concentrations. Moreover, it is confirmed that plant hormone signal transduction, amino acid metabolic and biosynthetic processes, plant-pathogen interaction as well as metal ion transport pathways were actively involved in the impact of PSNPs on wheat. In this regard, our findings would provide new insights into the wheat plant response to PSNPs exposure and could be of particularly great value to future research on the interaction of NPs and crop plants.

\section{Declarations}

\section{Author Contributions}

Q.X. Zhou and W.T. Liu conceived and designed the study. Q.X. Zhou, W.T. Liu and J.P. Lian interpreted the outcome of the experiments, and reviewed and extensively discussed the results. Q.X. Zhou, J.P. Lian and W.T. Liu wrote the manuscript with input from all authors. Q.X. Zhou and L. Q. Ma revised the manuscript. S.Z. Men performed GO and KEGG enrichment analysis. J.N. Wu and Y.B. Sun conducted seeds and chemicals purchase, plant cultivation and exposure. T.Z. Yang performed plant harvest and prepare. J.P. Lian and W.T. Liu performed total RNA isolation and libraries construction, mRNA sequence data processing, weighted gene co-expression network analysis, and validation of RNA-Seq by RT-qPCR.

\section{Competing interests}

All authors stated that they have no competing financial interests or personal relationships that could have appeared to influence the work reported in this paper.

\section{Acknowledgements}

This work was financially supported by the National Natural Science Foundation of China as joint key projects (U1906222 and U1806216) with the Shandong Provincial Government and as general projects (41471411 and 21677080), and the National Key Research and Development Program of China (2019YFC1804104 and 2020YFC180080-02).

\section{References}

1. Geyer R, Jambeck JR, Law KL. Production, use, and fate of all plastics ever made. Sci Adv 2017, 3(7): e1700782.

2. Barnes DKA, Galgani F, Thompson RC, Barlaz M. Accumulation and fragmentation of plastic debris in global environments. Philosophical Transactions Biological Sciences 2009, 364(1526): 1985-1998.

3. Rillig MC, Lehmann A. Microplastic in terrestrial ecosystems. Science 2020, 368(6498): 1430.

4. Rochman CM. Microplastics research-from sink to source. Science 2018, 360(6384): 28-29. 
5. Law KL, Thompson RC. Microplastics in the seas. Science 2014, 345(6193): 144-145.

6. Thompson RC, Olsen Y, Mitchell RP, Davis A, Rowland SJ, John AWG, et al. Lost at sea: where is all the plastic? Science 2004, 304(5672): 838.

7. Xu C, Zhang B, Gu C, Shen C, Yin S, Aamir M, et al. Are we underestimating the sources of microplastic pollution in terrestrial environment? J Hazard Mater 2020, 400: 123228.

8. de Souza Machado AA, Kloas W, Zarfl C, Hempel S, Rillig MC. Microplastics as an emerging threat to terrestrial ecosystems. Global Change Biol 2018, 24(4): 1405-1416.

9. Waldschläger K, Lechthaler S, Stauch G, Schüttrumpf H. The way of microplastic through the environment - Application of the source-pathway-receptor model (review). Sci Total Environ 2020, 713: 136584.

10. Talvitie J, Mikola A, Setälä O, Heinonen M, Koistinen A. How well is microlitter purified from wastewater? - A detailed study on the stepwise removal of microlitter in a tertiary level wastewater treatment plant. Water Res 2017, 109: 164-172.

11. Hernandez E, Nowack B, Mitrano D. Polyester textiles as a source of microplastics from households: A mechanistic study to understand microfiber release during washing. Environ Sci Techno/2017, 51(12): 7036-7046.

12. Ng E-L, Huerta Lwanga E, Eldridge SM, Johnston P, Hu H-W, Geissen V, et al. An overview of microplastic and nanoplastic pollution in agroecosystems. Sci Total Environ 2018, 627: 1377-1388.

13. Mahon AM, O'Connell B, Healy MG, O'Connor I, Officer R, Nash R, et al. Microplastics in sewage sludge: Effects of treatment. Environ Sci Technol 2017, 51(2): 810-818.

14. Du C-w, Zhou J-m, Shaviv A. Release Characteristics of Nutrients from Polymer-coated Compound Controlled Release Fertilizers. J Polym Environ 2006, 14(3): 223-230.

15. Qi Y, Yang X, Pelaez AM, Lwanga EH, Beriot N, Gertsen H, et al. Macro- and micro- plastics in soil-plant system: Effects of plastic mulch film residues on wheat (Triticum aestivum) growth. Sci Total Environ 2018, 645: 1048-1056.

16. Zhang D, Liu H-b, Hu W-I, Qin X-h, Ma X-w, Yan C-r, et al. The status and distribution characteristics of residual mulching film in Xinjiang, China. Journal of Integrative Agriculture 2016, 15(11): 2639-2646.

17. Liu K, Wang X, Song Z, Wei N, Li D. Terrestrial plants as a potential temporary sink of atmospheric microplastics during transport. Sci Total Environ 2020, 742: 140523.

18. Evangeliou N, Grythe H, Klimont Z, Heyes C, Eckhardt S, Lopez-Aparicio S, et al. Atmospheric transport is a major pathway of microplastics to remote regions. Nature Communications 2020, 11(1): 3381.

19. Zhang GS, Liu YF. The distribution of microplastics in soil aggregate fractions in southwestern China. Sci Total Environ 2018, 642: 12-20.

20. Scheurer M, Bigalke M. Microplastics in Swiss floodplain soils. Environ Sci Technol 2018, 52(6): 3591-3598. 
21. PiehI S, Leibner A, Löder MGJ, Dris R, Bogner C, Laforsch C. Identification and quantification of macro- and microplastics on an agricultural farmland. Scientific reports 2018, 8(1): 17950.

22. Hernandez LM, Xu EG, Larsson HCE, Tahara R, Maisuria VB, Tufenkji N. Plastic teabags release billions of microparticles and nanoparticles into tea. Environ Sci Technol 2019, 53(21): 12300-12310.

23. Editorial. Nanoplastic should be better understood. Nature nanotechnology 2019, 14(4): 299.

24. Taylor S, Pearce C, Sanguinet K, Hu D, Chrisler W, Kim Y-M, et al. Polystyrene Nano- and Microplastic Accumulation at Arabidopsis and Wheat Root Cap Cells, but No Evidence for Uptake into Roots. Environmental Science: Nano 2020, 7(7): 1942-1953.

25. Kalčíková G, Žgajnar Gotvajn A, Kladnik A, Jemec A. Impact of polyethylene microbeads on the floating freshwater plant duckweed Lemna minor. Environ Pollut 2017, 230: 1108-1115.

26. Zhang W, Dong Z, Zhu L, Hou Y, Qiu Y. Direct Observation of the Release of Nanoplastics from Commercially Recycled Plastics with Correlative Raman Imaging and Scanning Electron Microscopy. ACS Nano 2020, 14(7): 7920-7926.

27. Lian J, Wu J, Xiong H, Zeb A, Yang T, Su X, et al. Impact of polystyrene nanoplastics (PSNPs) on seed germination and seedling growth of wheat (Triticum aestivum L.). J Hazard Mater 2020, 385: 121620.

28. Mitrano DM, Beltzung A, Frehland S, Schmiedgruber M, Cingolani A, Schmidt F. Synthesis of metaldoped nanoplastics and their utility to investigate fate and behaviour in complex environmental systems. Nature nanotechnology 2019, 14(4): 362-368.

29. Rillig MC. Plastic and plants. Nature Sustainability 2020.

30. Zhou C-Q, Lu C-H, Mai L, Bao L-J, Liu L-Y, Zeng EY. Response of rice (Oryza sativa L.) roots to nanoplastic treatment at seedling stage. J Hazard Mater 2021, 401: 123412.

31. Wu X, Liu Y, Yin S, Xiao K, Xiong Q, Bian S, et al. Metabolomics revealing the response of rice (Oryza sativa L.) exposed to polystyrene microplastics. Environ Pollut 2020, 266: 115159.

32. Jiang $X$, Chen H, Liao Y, Ye Z, Li M, Klobučar G. Ecotoxicity and genotoxicity of polystyrene microplastics on higher plant Vicia faba. Environ Pollut 2019, 250: 831-838.

33. Boots B, Russell CW, Green DS. Effects of microplastics in soil ecosystems: above and below ground. Environ Sci Technol 2019, 53(19): 11496-11506.

34. de Souza Machado A, Abel, Lau CW, Kloas W, Bergmann J, Bachelier JB, Faltin E, et al. Microplastics can change soil properties and affect plant performance. Environ Sci Technol 2019, 53(10): 60446052.

35. Sun X-D, Yuan X-Z, Jia Y, Feng L-J, Zhu F-P, Dong S-S, et al. Differentially charged nanoplastics demonstrate distinct accumulation in Arabidopsis thaliana. Nature nanotechnology 2020, 15(9): 755-760.

36. Ahmad HR, Zia-ur-Rehman M, Sohail MI, Anwar ul Haq M, Khalid H, Ayub MA, et al. Chapter 11 Effects of Rare Earth Oxide Nanoparticles on Plants. In: Tripathi DK, Ahmad P, Sharma S, Chauhan 
DK, Dubey NK (eds). Nanomaterials in Plants, Algae, and Microorganisms. Academic Press, 2018, pp 239-275.

37. Weert Sv, E. R-HP, Diepens NJ, Koelmans AA. Effects of nanoplastics and microplastics on the growth of sediment-rooted macrophytes. Sci Total Environ 2019, 654: 1040-1047.

38. Bosker T, Bouwman LJ, Brun NR, Behrens P, Vijver MG. Microplastics accumulate on pores in seed capsule and delay germination and root growth of the terrestrial vascular plant Lepidium sativum. Chemosphere 2019, 226: 774-781.

39. Landa P, Dytrych P, Prerostova S, Petrova S, Vankova R, Vanek T. Transcriptomic Response of Arabidopsis thaliana Exposed to CuO Nanoparticles, Bulk Material, and lonic Copper. Environ Sci Technol 2017, 51(18): 10814-10824.

40. Landa P, Prerostova S, Petrova S, Knirsch V, Vankova R, Vanek T. The Transcriptomic Response of Arabidopsis thaliana to Zinc Oxide: A Comparison of the Impact of Nanoparticle, Bulk, and lonic Zinc. Environ Sci Technol 2015, 49(24): 14537-14545.

41. Trapnell C, Williams BA, Pertea G, Mortazavi A, Kwan G, van Baren MJ, et al. Transcript assembly and quantification by RNA-Seq reveals unannotated transcripts and isoform switching during cell differentiation. Nat Biotechnol 2010, 28(5): 511-515.

42. Hollender CA, Kang C, Darwish O, Geretz A, Matthews BF, Slovin J, et al. Floral Transcriptomes in Woodland Strawberry Uncover Developing Receptacle and Anther Gene Networks. Plant Physiol 2014, 165(3): 1062-1075.

43. Li L, Luo Y, Li R, Zhou Q, Peijnenburg WJGM, Yin N, et al. Effective uptake of submicrometre plastics by crop plants via a crack-entry mode. Nature Sustainability 2020.

44. Qi Y, Beriot N, Gort G, Huerta Lwanga E, Gooren H, Yang X, et al. Impact of plastic mulch film debris on soil physicochemical and hydrological properties. Environ Pollut 2020, 266: 115097.

45. Rillig MC, Lehmann A, de Souza Machado AA, Yang G. Microplastic effects on plants. New Phytol 2019, 223: 1066-1070.

46. Yu LJ, Luo YF, Liao B, Xie LJ, Chen L, Xiao S, et al. Comparative transcriptome analysis of transporters, phytohormone and lipid metabolism pathways in response to arsenic stress in rice (Oryza sativa). New Phytol 2012, 195(1): 97-112.

47. Urbina MA, Correa F, Aburto F, Ferrio JP. Adsorption of polyethylene microbeads and physiological effects on hydroponic maize. Sci Total Environ 2020, 741: 140216.

48. Yan B, Ji Z, Fan B, Wang X, He G, Shi L, et al. Plants adapted to nutrient limitation allocate less biomass into stems in an arid-hot grassland. New Phytol 2016, 211(4): 1232-1240.

49. Shaul O. Magnesium transport and function in plants: the tip of the iceberg. BioMetals 2002, 15(3): 307-321.

50. Kuhn AJ, Schröder WH, Bauch J. The kinetics of calcium and magnesium entry into mycorrhizal spruce roots. Planta 2000, 210(3): 488-496. 
51. Aibara I, Miwa K. Strategies for optimization of mineral nutrient transport in plants: multilevel regulation of nutrient-dependent dynamics of root architecture and transporter activity. Plant Cell Physiol 2014, 55(12): 2027-2036.

52. Wang M, Zechen G, Wang R, Guo J, Ling N, Firbank L, et al. Plant Primary Metabolism Regulated by Nitrogen Contributes to Plant-Pathogen Interactions. Plant Cell Physiol 2018, 60(2): 329-342.

53. Schwab F, Rothen-Rutishauser B, Petri-Fink A. When plants and plastic interact. Nature Nanotechnology 2020, 15(9): 729-730.

\section{Table}

Table 1. KEGG pathway classification of transcripts based on MetaDE and WGCNA analysis. 


\begin{tabular}{|c|c|c|c|c|c|}
\hline \multicolumn{2}{|c|}{ Treatment } & \multirow{2}{*}{$\begin{array}{l}\text { KEGG ID } \\
\text { ko01200 }\end{array}$} & \multirow{2}{*}{$\begin{array}{l}\text { Pathway category } \\
\text { Carbon metabolism }\end{array}$} & \multirow{2}{*}{$\begin{array}{l}\text { P-value } \\
1.28 \mathrm{E}- \\
04\end{array}$} & \multirow{2}{*}{$\begin{array}{l}\begin{array}{l}\text { Gene } \\
\text { count }\end{array} \\
49 \\
(10.3 \%)\end{array}$} \\
\hline WGCNA & $\begin{array}{l}\text { MEblue- } \\
\text { green }\end{array}$ & & & & \\
\hline & & ko01230 & Biosynthesis of amino acids & $\begin{array}{l}3.64 \mathrm{E}- \\
04\end{array}$ & $47(9.9 \%)$ \\
\hline & & ko00030 & Pentose phosphate pathway & $\begin{array}{l}7.57 \mathrm{E}- \\
03\end{array}$ & $13(2.7 \%)$ \\
\hline & & ko00190 & Oxidative phosphorylation & $\begin{array}{l}7.57 \mathrm{E}- \\
03\end{array}$ & $20(4.2 \%)$ \\
\hline & & ko00260 & $\begin{array}{l}\text { Glycine, serine and threonine } \\
\text { metabolism }\end{array}$ & $\begin{array}{l}8.20 \mathrm{E}- \\
03\end{array}$ & $14(3.0 \%)$ \\
\hline & & ko00360 & Phenylalanine metabolism & $\begin{array}{l}8.20 \mathrm{E}- \\
03\end{array}$ & $9(1.9 \%)$ \\
\hline & $\begin{array}{l}\text { Meturquo.- } \\
\text { red }\end{array}$ & ko03010 & Ribosome & $\begin{array}{l}3.19 \mathrm{E}- \\
15\end{array}$ & $\begin{array}{l}87 \\
(16.3 \%)\end{array}$ \\
\hline & & ko01200 & Carbon metabolism & $\begin{array}{l}7.56 \mathrm{E}- \\
03\end{array}$ & $47(8.8 \%)$ \\
\hline \multirow[t]{10}{*}{ MetaDE } & Shoot & ko04016 & MAPK signaling pathway - plant & $\begin{array}{l}2.84 \mathrm{E}- \\
04\end{array}$ & $35(5.3 \%)$ \\
\hline & & ko04075 & Plant hormone signal transduction & $\begin{array}{l}6.55 \mathrm{E}- \\
04\end{array}$ & $50(7.6 \%)$ \\
\hline & & ko00564 & Glycerophospholipid metabolism & $\begin{array}{l}3.92 \mathrm{E}- \\
03\end{array}$ & $24(3.7 \%)$ \\
\hline & & ko04626 & Plant-pathogen interaction & $\begin{array}{l}3.92 \mathrm{E}- \\
03\end{array}$ & $39(5.9 \%)$ \\
\hline & Root & ko04626 & Plant-pathogen interaction & $\begin{array}{l}5.14 \mathrm{E}- \\
06\end{array}$ & $46(7.4 \%)$ \\
\hline & & ko04075 & Plant hormone signal transduction & $\begin{array}{l}5.62 \mathrm{E}- \\
05\end{array}$ & $51(8.2 \%)$ \\
\hline & & ko04016 & MAPK signaling pathway - plant & $\begin{array}{l}7.98 \mathrm{E}- \\
05\end{array}$ & $34(5.5 \%)$ \\
\hline & & ko00564 & Glycerophospholipid metabolism & $\begin{array}{l}1.31 \mathrm{E}- \\
03\end{array}$ & $24(3.9 \%)$ \\
\hline & & ko00940 & Phenylpropanoid biosynthesis & $\begin{array}{l}2.72 \mathrm{E}- \\
03\end{array}$ & $30(4.8 \%)$ \\
\hline & & ko00561 & Glycerolipid metabolism & $\begin{array}{l}8.31 \mathrm{E}- \\
03\end{array}$ & $17(2.7 \%)$ \\
\hline
\end{tabular}




\section{Figures}

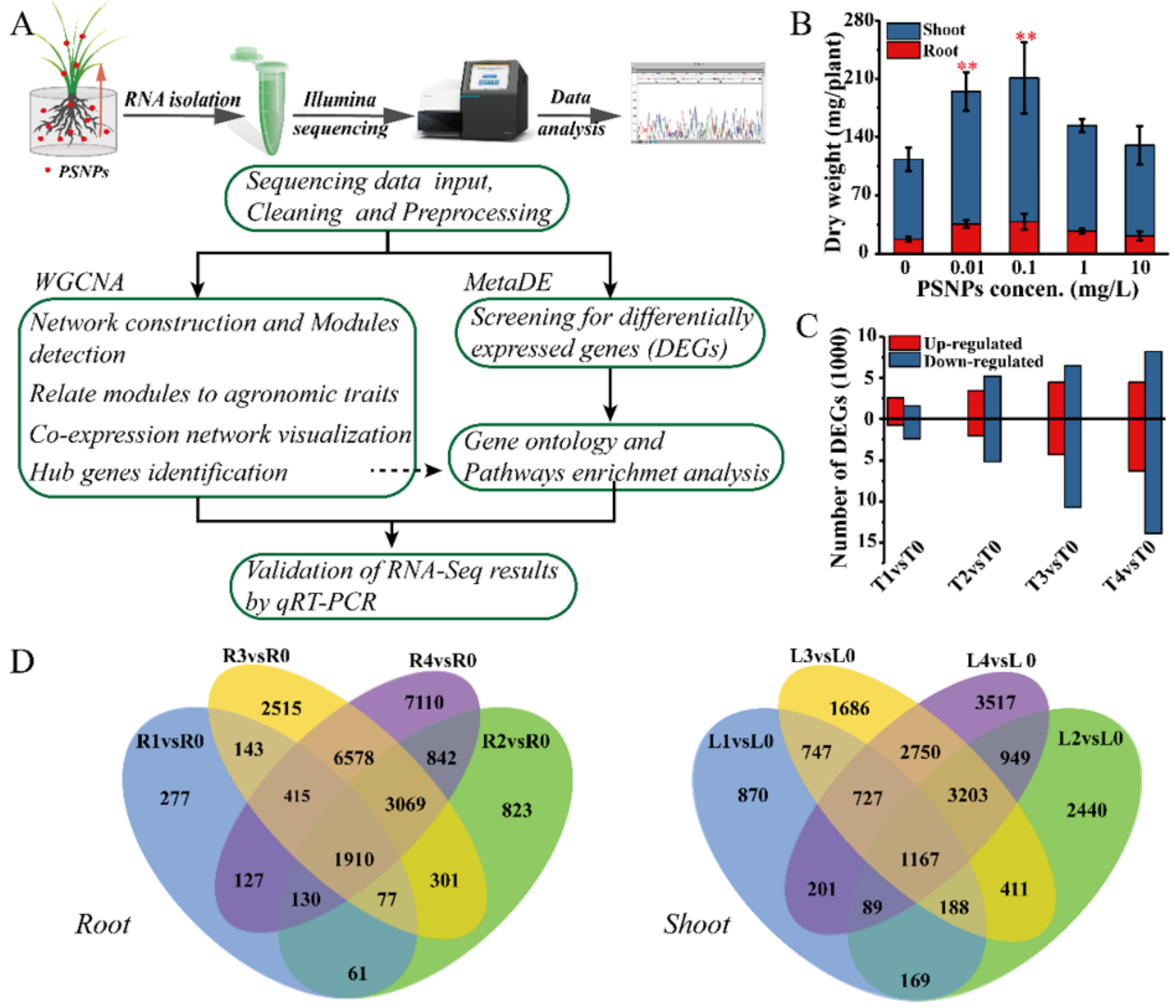

\section{Figure 1}

Experimental design for gene detection in PSNPs exposed wheat seedlings after cultured in hydroponic medium for three weeks. (A) Schematic diagram of wheat total RNA isolation, sequencing, and corresponding data processing processes. (B) Average dry weight of wheat root and shoot $(n=4)$ when exposed to different PSNPs concentrations (0-10 mg/L). Data were represented as mean \pm standard deviation with a statistical significance less than 0.05 (Duncan's post hoc test). Asterisk ** indicates that $P<0.01$. (C) The distribution of differentially expressed genes (DEGs) number in wheat roots and shoots 
among four PSNPs treatments. Parts below the X-axis represent wheat root, while the above parts are wheat shoots. (D) Venn diagram for the DEGs expression variation among different PSNPs treatment in root and shoot, respectively.

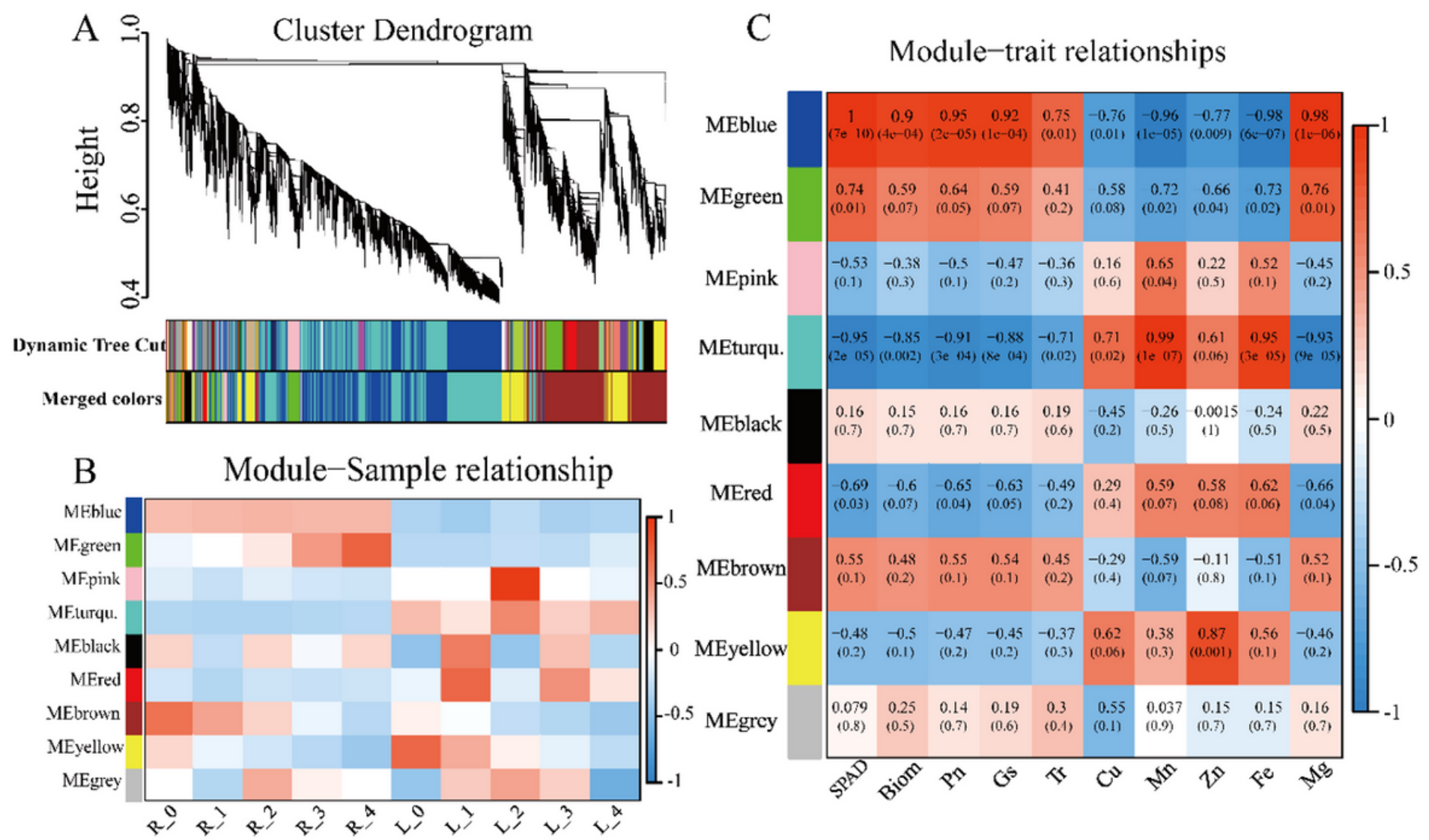

\section{Figure 2}

Network module identification and module correlation analysis. (A) Cluster dendrogram and eigengenes distribution in the obtained modules. (B) Modules' eigengenes (MEs) correlated with wheat tissue samples. Red and blue color of each grid mean the positive and negative correlation of sample with MEs, respectively. (C) Correlation between MEs and wheat traits after exposure to PSNPs for three weeks. Upper number in each grid represents the correlation coefficient, while the lower number is the p-value of statistical significance. 


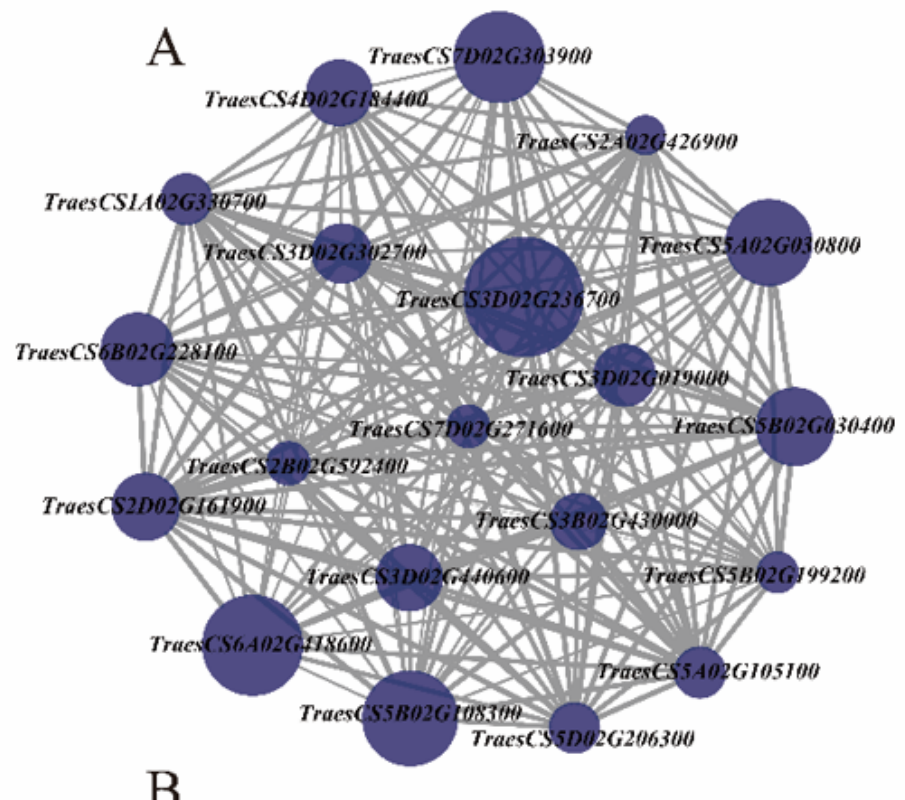

B

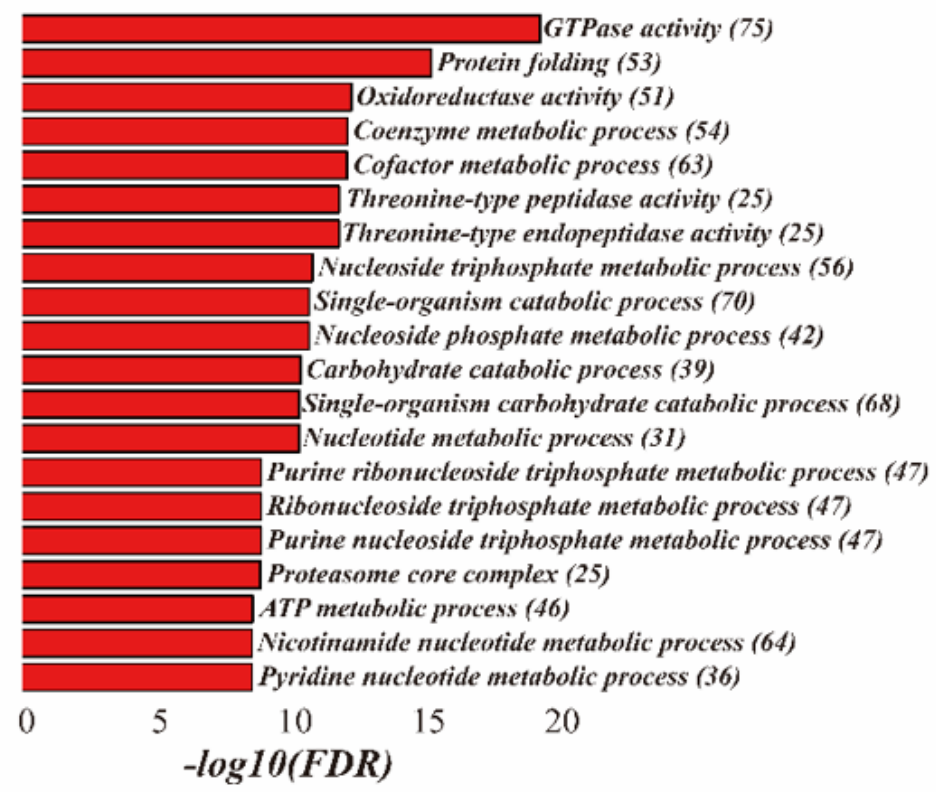

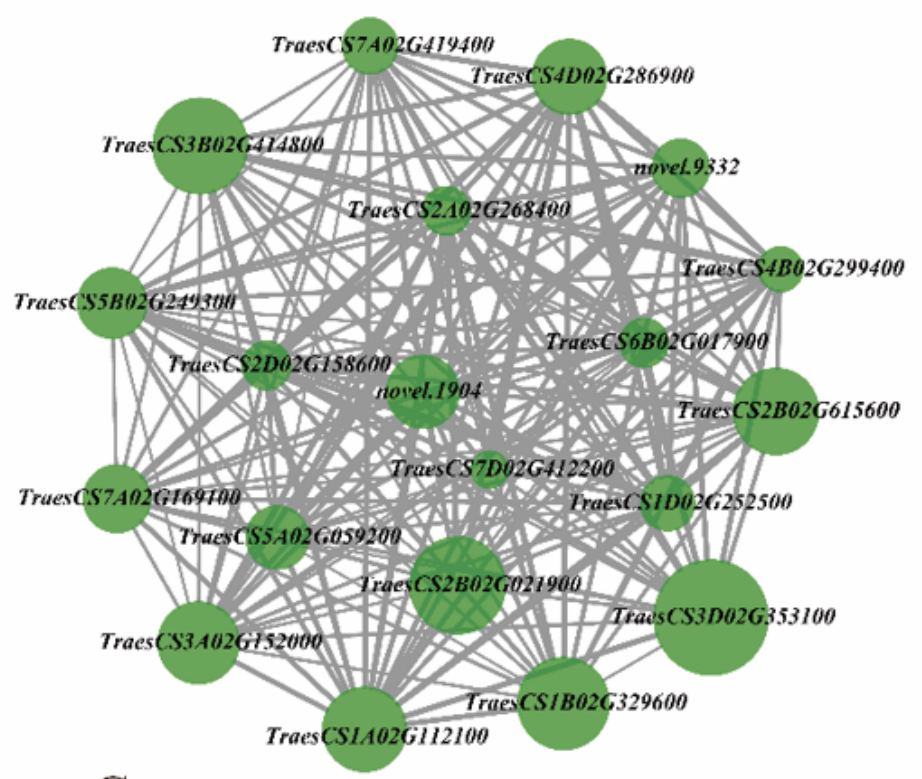

$\mathrm{C}$

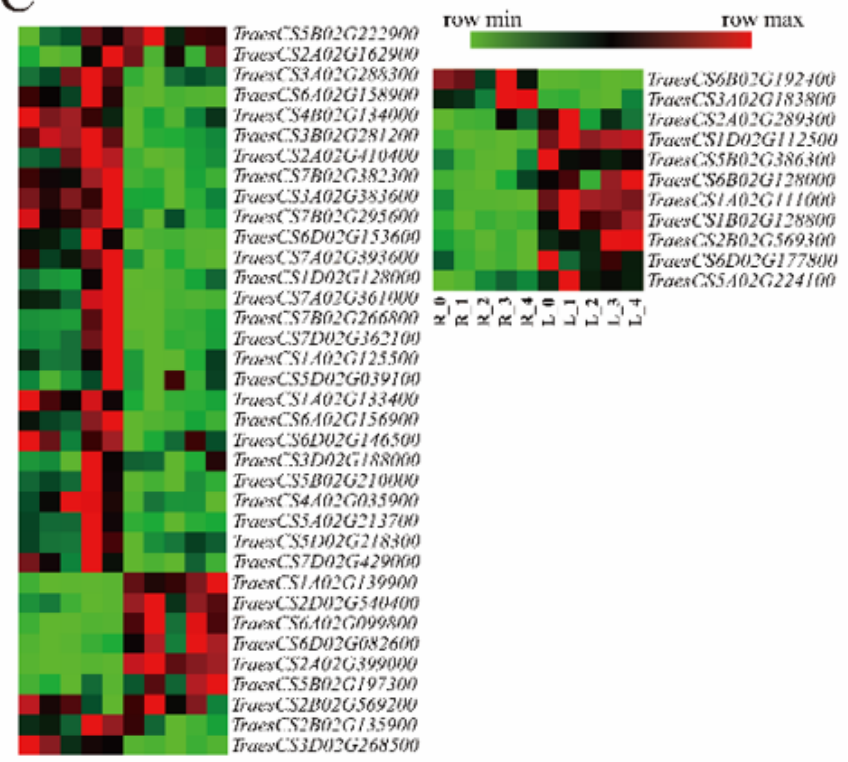

\section{Figure 3}

Modules positively correlated with plant performance, metabolites and macronutrient (Mg). (A) Top 20 most connected genes in MEblue and MEgreen are shown in their color assigned through co-expression network construction. The larger node size represents the higher connectivity of other genes in this module, and line thickness between nodes indicates the connection strength of two conjoint genes. (B) Top 20 most enriched GO terms obtained from the merge of eigengenes in MEblue and MEgreen. (C) Heatmap of genes expression associated with metal ion transport in wheat root and leaf samples. Relative expression of these genes are assigned with green to red gradient color showing the minimum and maximum value. 

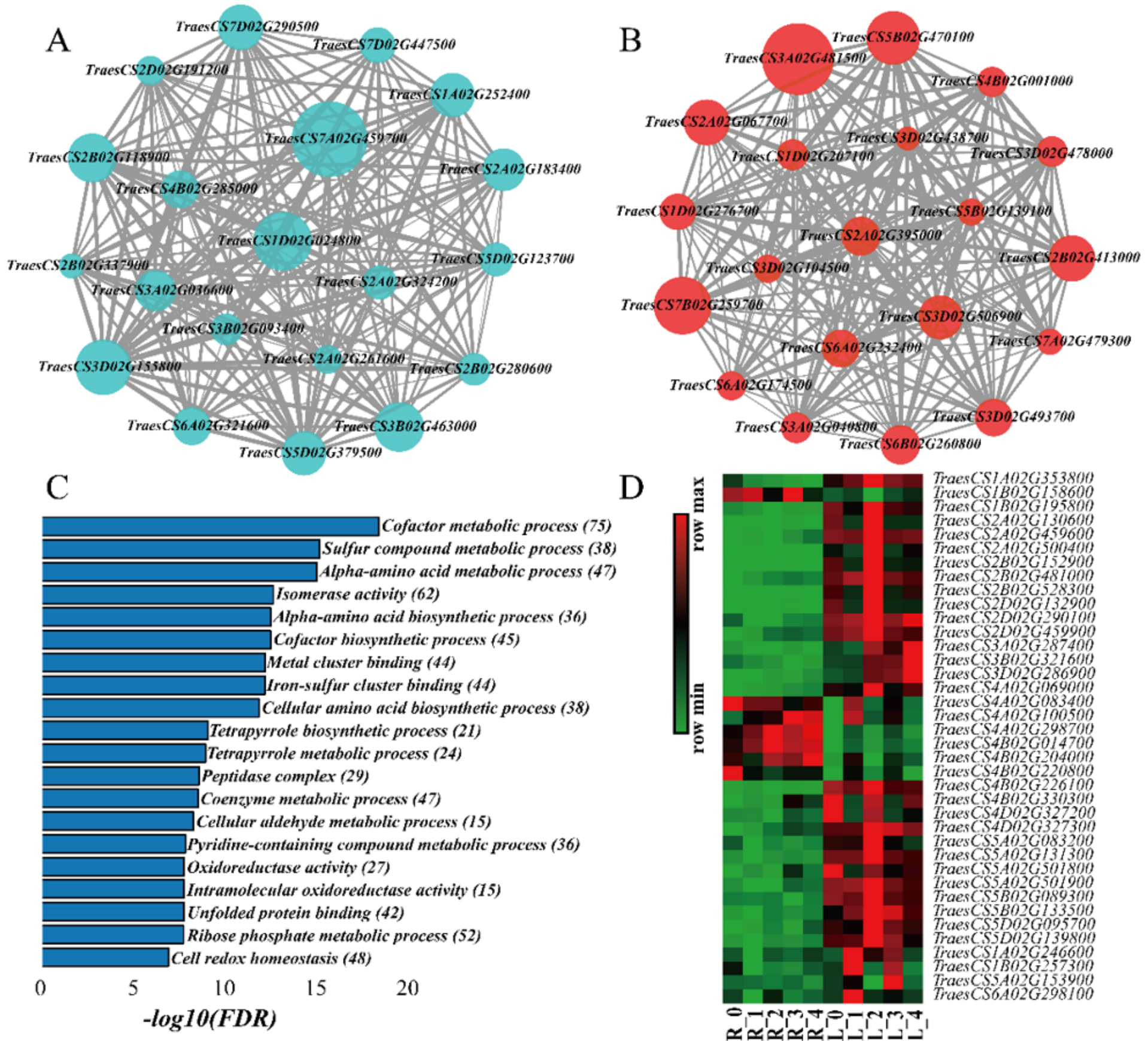

$\mathrm{D}$

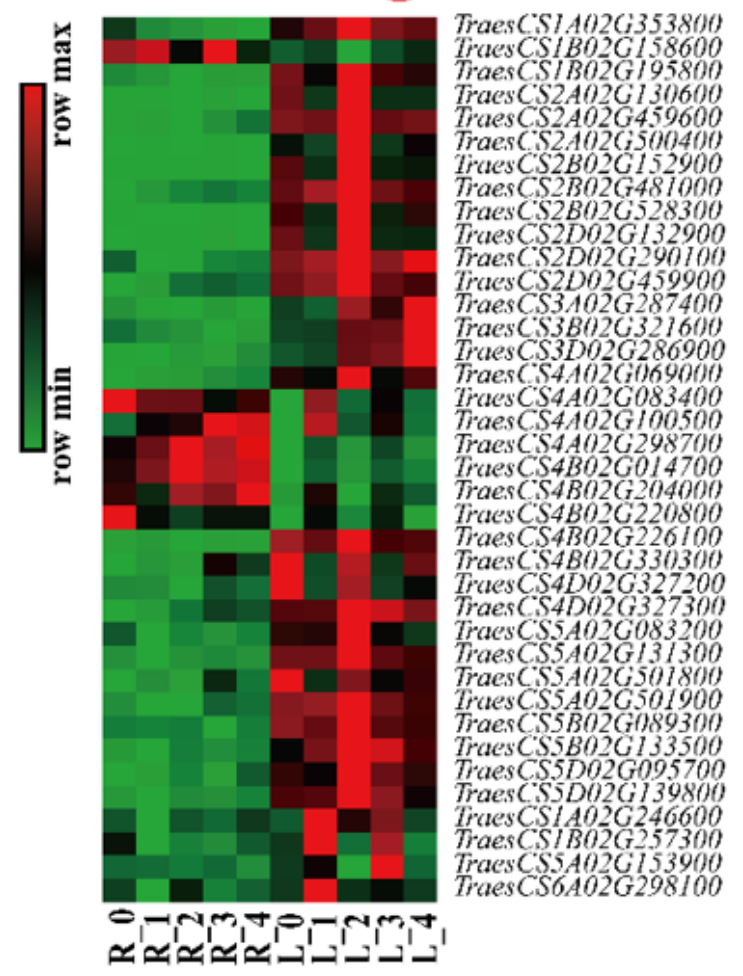

Figure 4

Modules negatively correlated with plant performance, metabolites and micronutrients. (A) Top 20 most connected genes in MEturquoise and MEred display in color assigned through co-expression network construction. The larger node size represents the higher connectivity of other genes in this module, and line thickness between nodes indicates the connection strength of two conjoint genes. (B) Top 20 most enriched GO terms obtained from the merge of eigengenes in MEturquoise and MEred. (C) Heatmap of genes expression associated with amino acid metabolic process in wheat root and leaf samples. Relative expression of these genes are assigned with green to red gradient color showing the minimum and maximum value. 


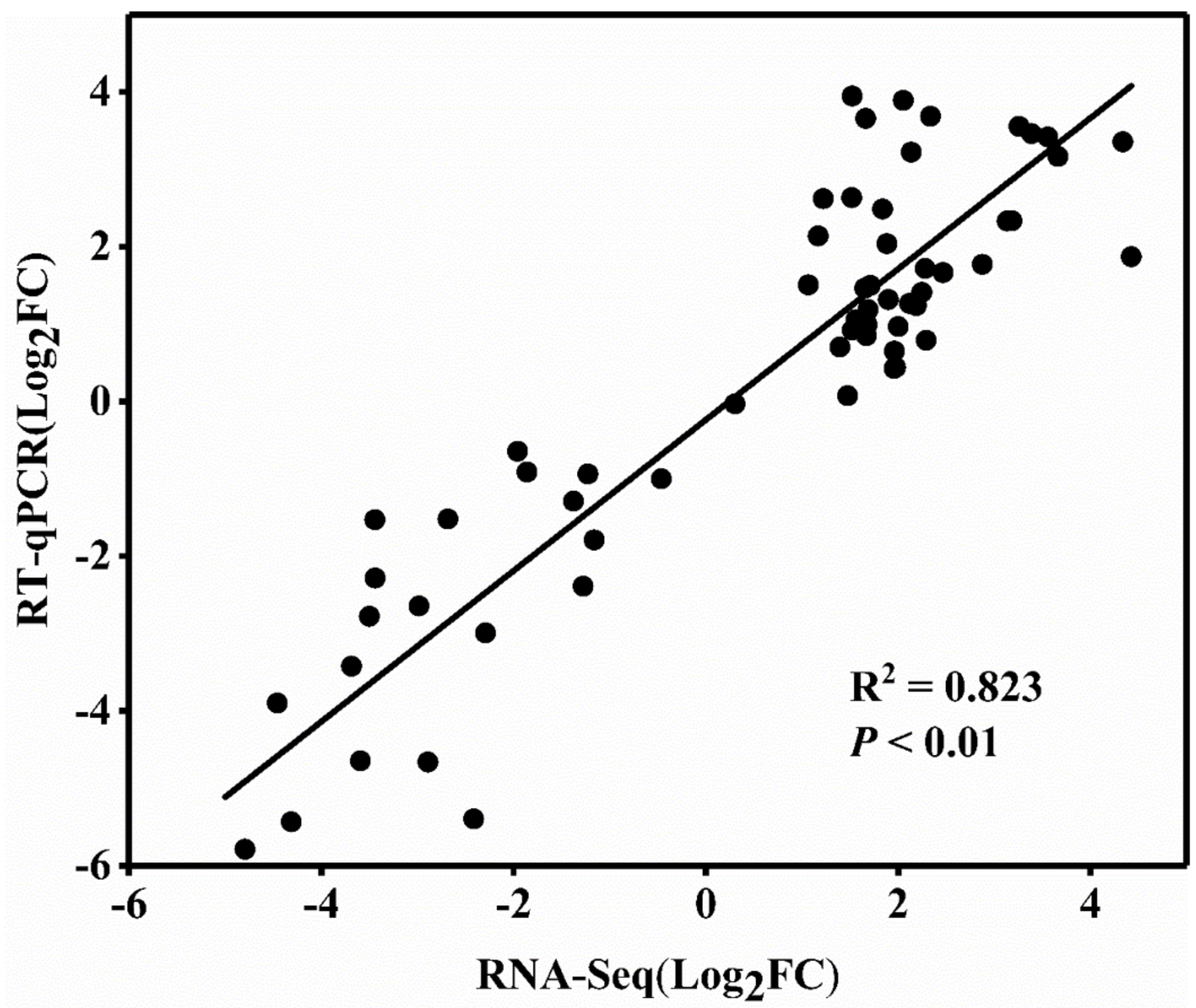

Figure 5

Ten selected genes were validated by RT-qPCR method comparatively, and linear correlation between RNA-seq and RT-qPCR data was presented with a log2 fold change transformation.

\section{Supplementary Files}

This is a list of supplementary files associated with this preprint. Click to download.

- Supplementaryinformation.docx 